\title{
Natural van der Waals heterostructure cylindrite with highly anisotropic optical responses
}

\author{
Arindam Dasgupta $\mathbb{D D}^{1}$, Jie Gao $\mathbb{D}^{1 凶}$ and Xiaodong Yang $\mathbb{D}^{1 凶}$
}

The mechanical exfoliation of naturally occurring layered materials has emerged as an easy and effective method for achieving ultrathin van der Waals (vdW) heterostructures with well-defined lattice orientations of the constituent two-dimensional (2D) material layers. Cylindrite is one such naturally occurring vdW heterostructure, where the superlattice is composed of alternating stacks of $\mathrm{SnS}_{2}$-like and PbS-like layers. Although the constituent 2D lattices are isotropic, inhomogeneous strain occurring from local atomic alignment for forcing the commensuration makes the cylindrite superlattice structurally anisotropic. Here, we demonstrate the highly anisotropic optical responses of cylindrite thin flakes induced by the anisotropic crystal structure, including angleresolved polarized Raman scattering, linear dichroism, and polarization-dependent anisotropic third-harmonic generation. Our results provide a promising approach for identifying various natural vdW heterostructure-based 2D materials with tailored optical properties and can be harnessed for realizing anisotropic optical devices for on-chip photonic circuits and optical information processing.

npj 2D Materials and Applications (2021)5:74; https://doi.org/10.1038/s41699-021-00254-9

\section{INTRODUCTION}

Since the discovery of graphene, van der Waals (vdW) materials are the subject of intensive research due to their fascinating optical, electrical, and mechanical properties. Hexagonal boron nitride, black phosphorus (BP), and transition metal dichalcogenides are a few examples among the other vdW materials, which have received great attention not only in the context of fundamental science but also for various practical applications ${ }^{1-3}$. In these materials, individual layers of chemically bonded atoms are stacked and held by vdW interactions, and the nature of the vdW stacking is critical in defining their physical properties. One of the most promising prospects is to design vdW heterostructures with distinct stacking symmetries and functionalities by reassembling individual atomic layers of different materials in a precisely chosen layer-by-layer sequence, where the electronic band structures, carrier mobility, optical and magnetic properties can be tailored ${ }^{4-9}$. The fabricated vdW heterostructures can enable intriguing functionalities beyond the reach of the existing materials and advance various photonic and optoelectronic applications covering a broad spectral range from visible to mid-IR for optical communication and sensing ${ }^{10}$, transistors $^{11,12}$, photodetectors ${ }^{13-15}$, and ultrafast lasers ${ }^{16}$.

Currently, there are two ways of fabricating vdW heterostructures. One approach is to directly grow them through layer-bylayer sequential synthesis using chemical vapor deposition $(C V D)^{17-19}$. However, this is a challenging task and it has only been demonstrated for a few vdW heterostructures so far. Another approach is to mechanically exfoliate the individual layers of different materials and then deterministically place one layer on top of the other ${ }^{20-23}$. Improper crystalline alignment between the stacked lattices and the presence of interlayer contaminants are two major issues for this method, which will affect the device performance. Alternatively, the mechanical exfoliation of naturally occurring vdW superlattices facilitates an easy and hassle-free approach for fabricating ultrathin layers of vdW heterostructures with well-defined lattice orientations of the individual layers. These materials are naturally formed with alternating layers of two different 2D materials due to the phase segregation process during their formation, and thus are free of the aforementioned issues. Several exfoliated naturally occurring vdW heterostructure materials such as cylindrite ${ }^{24}$, franckeite ${ }^{25}$, and levyclaudite ${ }^{26}$ have been reported, which belong to the sulfosalt mineral family with the vdW superlattices formed by alternate stacking of $\mathrm{SnS}_{2}$-like pseudo-hexagonal $(\mathrm{H})$ layer and $\mathrm{PbS}$-like pseudo-tetragonal $(\mathrm{Q})$ layer. Out of these materials, the optical and electrical properties of bulk to single unit cell thick franckeite have been explored for optoelectronic applications in the linear optical regime ${ }^{27,28}$. The high electrical conductivity and magnetic properties of cylindrite flakes have also been demonstrated ${ }^{24}$. Although the stacked alternating layers are comprised of isotropic vdW materials, the incommensurability between the constituent $\mathrm{SnS}_{2}$-like and PbSlike lattices introduces a strain-induced deformation in the crystals and hence induces the structural anisotropy showing anisotropic electrical, vibrational, and optical properties. The anisotropic optical properties of franckeite have recently been reported ${ }^{27}$. However, to date, there is no study on the anisotropic optical responses of cylindrite yet.

The recent demonstration of broadband nonlinear optical response with ultrafast carrier dynamics exhibited in franckeite has opened up the possibility of developing nonlinear optoelectronic devices based on naturally occurring vdW heterostructures $^{29}$. Nonetheless, the effect of the crystal structural anisotropy on the nonlinear harmonic generation in franckeite and cylindrite has not yet been explored. The optical anisotropy in vdW materials exhibits polarization-sensitive nonlinear light-matter interactions at the nanoscale, which is important in the context of many applications in optical information processing and communication $^{30}$. The ultrathin thicknesses of the exfoliated vdW materials make them appealing for realizing highperformance integrated nonlinear optical devices 31,32 . Previously, several types of vdW materials such as $\mathrm{BP}^{33-35}, \mathrm{ReS}_{2}{ }^{36}, \mathrm{GeSe}^{37}$, and $\mathrm{GeAs}^{38}$ have been explored for anisotropic third-harmonic generation (THG). The widely-used anisotropic nonlinear 2D

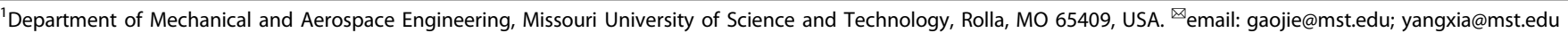



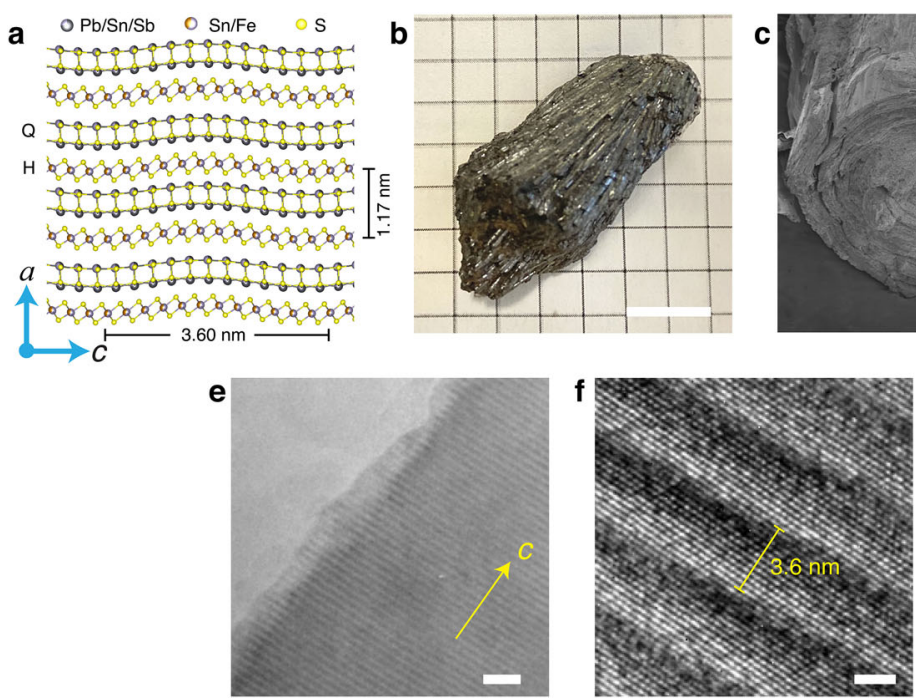
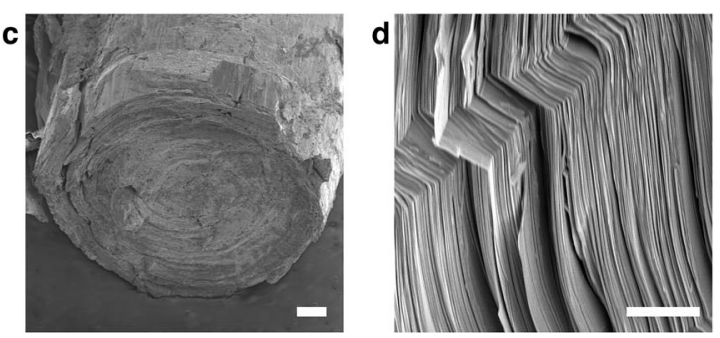

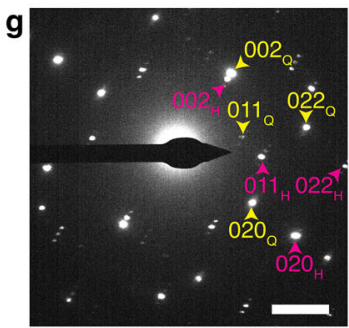

Fig. 1 Morphology and crystal structure of cylindrite. a Schematic diagram of the crystal structure of cylindrite composed of alternating $\mathrm{SnS}_{2}$-like pseudo-hexagonal layer (H-layer) and PbS-like pseudo-tetragonal layer (Q-layer). b Optical image of the cylindrite mineral rock. The scale bar is $10 \mathrm{~mm}$. c SEM image of the cross-section of an individual cylindrite cylinder showing the crystals are formed by concentric layers of materials held by vdW interactions. The scale bar is $100 \mu \mathrm{m}$. d Zoomed-in SEM image of a region around the revolution axis showing the concentric layers. The scale bar is $5 \mu \mathrm{m}$. e TEM image of a mechanically exfoliated ultrathin cylindrite flake showing the characteristic striped patterns along the $c$-direction of the crystal, caused by the out-of-plane rippling due to the forced commensuration of the constituent $\mathrm{H}$ - and Q-layers. The scale bar is $10 \mathrm{~nm}$. f Representative HRTEM image showing the atomic arrangements. The scale bar is $2 \mathrm{~nm}$. $\mathbf{g}$ Corresponding SAED pattern consisting with diffraction spots related to H-layer (magenta) and Q-layer (yellow). The scale bar is $2 \mathrm{~nm}^{-1}$.

material of BP shows strong third-order optical nonlinearity, but BP is unstable under ambient conditions which limit its use in practical applications. Although other materials of $\mathrm{ReS}_{2}, \mathrm{GeSe}$, and GeAs are stable, they exhibit simple crystal structures formed by stacking only one type of molecular layer. Additionally, nonlinear harmonic generation is also regarded as a rapid and reliable characterization tool for evaluating material homogeneity and identifying crystal orientation and thickness of 2D materials ${ }^{39,40}$.

Here, we study the highly anisotropic optical responses of mechanically exfoliated cylindrite thin flakes down to a few unit cell thickness. First, we perform transmission electron microscopy (TEM), energy-dispersive X-ray spectroscopy (EDXS), and X-ray photoelectron spectroscopy (XPS) analysis to determine the anisotropic crystal structure of cylindrite and its chemical composition. We also demonstrate that the in-plane structural anisotropy of cylindrite flakes can be revealed by angle-resolved polarized Raman spectroscopy. Furthermore, the optical anisotropy induced linear dichroism in cylindrite flakes is shown by performing polarization-dependent differential reflectance spectroscopy (DRS) measurements. Finally, highly anisotropic THG from cylindrite flakes is demonstrated by characterizing the angular dependence of THG emission with respect to the pump polarization. The third-order nonlinear susceptibility of cylindrite is also estimated to be in the same order of magnitude as other anisotropic nonlinear 2D materials. The demonstrated results are promising for identifying various natural vdW heterostructures to expand the current existing 2D material library. Our results can also be harnessed further to realize ultrathin anisotropic optical devices for polarization-sensitive photodetectors, optical modulators, frequency- and polarization-multiplexed encoding prototypes for secure optical information processing, and communication.

\section{RESULTS}

\section{Morphology and chemical composition of cylindrite}

Cylindrite was first discovered by Frenzel from a tin-mining region in Bolivia in 1893. It attracted attention due to its peculiar triclinic crystal structure with a tendency to develop large concentric cylindrical shells and aggregates. Later, wet chemical analysis established that cylindrite belongs to the sulfosalt mineral family and has a generic chemical formula $\mathrm{Pb}_{3} \mathrm{Sn}_{4} \mathrm{FeSb}_{2} \mathrm{~S}_{14}{ }^{41}$. Artificial synthesis of the cylindrite structures with user-tailored composition has also been achieved ${ }^{42,43}$. Recently, liquid-phase and mechanical exfoliation of cylindrite thin flakes down to a few nanometers thick have been reported, and it is shown that cylindrite flakes exhibit a narrow bandgap $<0.85 \mathrm{eV}$ and are stable under ambient conditions ${ }^{24}$. Figure $1 \mathrm{a}$ is a simplified representation of the cylindrite crystal structure, indicating alternative threeatom-thick $\mathrm{SnS}_{2}$-like layer (H-layer) and two-atom-thick PbS-like layer (Q-layer) bonded together via vdW forces. The optical image in Fig. $1 \mathrm{~b}$ shows the characteristic appearance of a cylindrite mineral rock containing clusters of long cylinders with gray-black color. Figure1c is a scanning electron microscope (SEM) image of the cross-section of an individual cylindrite cylinder, which shows that the crystals are formed by concentric layers of materials held by vdW interactions. The zoomed-in view in Fig. 1d illustrating a region around the revolution axis of the cylinder justifies its cylindrical shape. The following notation is adopted to describe the crystal structure of cylindrite, as [100] (a-axis) is considered to be the out-of-plane direction which is the direction of layer stacking, while [010] (b-axis) and [001] (c-axis) are considered for the two in-plane directions. Also, $a, \beta$, and $\gamma$ are the angles between the $a, b$, and $c_{0}$ axis. The unit cell parameters of the $\mathrm{H}$-layer are $a_{\mathrm{H}}=11.709 \mathrm{~A}, b_{\mathrm{H}}=3.670 \mathrm{~A}, c_{\mathrm{H}}=6.320 \mathrm{~A}, a_{\mathrm{H}}=90^{\circ}$, $\beta_{H}=92.85^{\circ}$, and $\gamma_{H}=90,58^{\circ}$, while the lattice parameters for the Q-layer are $a_{\mathrm{Q}}=11.733 \mathrm{~A}, b_{\mathrm{Q}}=5.790 \mathrm{~A}, c_{\mathrm{Q}}=5.810 \mathrm{~A}, a_{\mathrm{Q}}=90^{\circ}$, $\beta_{\mathrm{Q}}=92.38^{\circ}$, and $\gamma_{\mathrm{Q}}=93.87^{\circ 41}$. Due to the small mismatch of the stacking vectors $\left(a_{\mathrm{H}}\right.$ and $\left.a_{\mathrm{Q}}\right)$ and their corresponding $\beta$ and $\gamma$ values, the coincidence cell or a supercell contains 12 layer pairs with $a=140$ A. Furthermore, the lattice mismatch in the $b$ and $c$ directions indicates that the supercell is made of 30:19 and 12:13 unit cells of $\mathrm{H}$ - and Q-type unit cells in these two directions, respectively. The inhomogeneous elastic deformation and local atomic alignment caused to force the commensuration between incommensurate $\mathrm{H}$ - and Q-layers will result in the interlayer moire patterns in vdW heterostructures ${ }^{24,25,27,28}$. As the crystal relaxes to minimize the total energy, strong in-plane strain is developed to form small out-of-plane rippling modulated perpendicular to the 
moiŕe pattern, resulting in a strong in-plane structural anisotropy in the crystal. Figure 1a schematically illustrates the rippling in the cylindrite crystal lattice. The TEM image of a mechanically exfoliated cylindrite thin flake transferred onto a TEM copper grid (details of the TEM sample preparation is included in the Methods section) in Fig. 1e clearly shows an in-plane striped pattern of periodic darker and lighter areas arising from the rippling on the surface of cylindrite flake along the c-direction. The highresolution (HR) TEM image in Fig. if shows the atomic arrangements of $\mathrm{H}$ - and Q-layers in a cylindrite flake. The fringes occurring from the rippling along the $c$-direction are spaced with a period of $3.6 \mathrm{~nm}$. Although the constituent $\mathrm{H}$ - and Q-lattices are comprised of isotropic vdW materials, the incommensurability between them introduces a strain-induced deformation in the crystals and hence induces the structural anisotropy in the heterostructure superlattice. Therefore, the interlayer moiŕe patterns observed in the TEM images confirm the anisotropic heterostructure of cylindrite with strain-induced deformation. The selected area diffraction pattern (SAED) from the crystal normal to the [100] crystal zone axis is shown in Fig. 1g. The SAED exhibits the reflections from the corresponding $\mathrm{H}$ - and Q-layers, which are marked in magenta and yellow colors, respectively. Two distinct sets of reflection spots in rectangular arrangements indicate different $b$ and $c$ unit cell parameters for the constituent $\mathrm{H}$ - and Qlayers, which manifests the heterostructure superlattice is composed of two alternatively stacked incommensurate 2D lattices with strain-induced deformation.

EDXS analysis is performed to analyze the chemical composition of the exfoliated cylindrite flakes. The averaged EDXS spectrum in Fig. 2a acquired from a thin cylindrite flake shows the presence of main elements $\mathrm{Pb}, \mathrm{Sn}, \mathrm{Sb}, \mathrm{Fe}$, and $\mathrm{S}$. Other elements such as $\mathrm{C}, \mathrm{O}$, and $\mathrm{Si}$ are also detected, which is expected for naturally occurring minerals where other elements can also coexist. However, the signal of $\mathrm{Cu}$ is present due to the copper TEM grid. Most of the signal of $\mathrm{C}, \mathrm{O}$, and $\mathrm{Si}$ may also come from the underlying carbon film support of the TEM grid, and the accumulation of carbonbased adsorbates during the transfer of the flakes on the grid (see Supplementary Figure 1). Figure $2 b-f$ plot the TEM-EDXS elemental maps of the main elements $\mathrm{Pb}, \mathrm{Sn}, \mathrm{Sb}, \mathrm{Fe}$, and $\mathrm{S}$ from the area of a cylindrite crystal shown in the bright-field (BF)-TEM image in the inset of Fig. 2a. These maps show that all the elements are homogeneously distributed in the crystal when viewed from the top along [100] direction of the crystal. The quantification of each element in the crystal is listed in Table 1, from which the approximate chemical formula of the cylindrite flake under study is determined to be $\mathrm{Pb}_{2.9} \mathrm{Sn}_{4.0} \mathrm{Fe}_{0.9} \mathrm{Sb}_{1.9} \mathrm{~S}_{14}$. This approximate chemical formula is not completely charge-balanced which is mainly caused by the low sensitivity of EDXS, the overlap between $\mathrm{S} \mathrm{K}_{\alpha}$ and $\mathrm{Pb} \mathrm{M}_{\alpha}$ peaks, as well as the overlap between $\mathrm{Sn}$ $\mathrm{L}_{\beta}$ and $\mathrm{Sb} \mathrm{L}_{\alpha}$ peaks.

\section{Surface chemical composition of cylindrite}

The surface chemical composition including the effect of oxidation on the surface of air-aged cylindrite is explored using XPS since the penetration depth of the XPS technique is limited to a few nanometers. The cylindrite crystal is kept under ambient conditions for three weeks after cleaving it out of the cylindrite rock. Figure $2 \mathrm{~g}$ shows the XPS spectrum of air-aged cylindrite, which is used for the quantification of the main elements in Table 2. The approximate surface chemical formula of cylindrite obtained from the compositional stoichiometry analysis is $\mathrm{Pb}_{3.5} \mathrm{Sn}_{4.0} \mathrm{Fe}_{0.9} \mathrm{Sb}_{1.9} \mathrm{~S}_{14}$. In comparison to EDXS, a slightly larger concentration of $\mathrm{Pb}$ is found in the derived stoichiometry from the XPS survey spectra. Although the obtained stoichiometry is very close to the ideal formula of cylindrite, there is the possibility of slight degradation or shear deformation of the surface while performing the exfoliation. Further, the oxidation states of individual elements are determined by the peak fitting in the high-resolution spectra at $\mathrm{Pb} 4 f, \mathrm{Sn} 3 d, \mathrm{Sb}$ $3 d$, Fe $2 p$, and $\mathrm{S} 2 p$ binding energy regions, as presented in Fig. $2 \mathrm{~h}$ l. As shown in Fig. $2 \mathrm{~h}$, lead is present in the material as $\mathrm{Pb}^{2+}(88 \%)$ and $\mathrm{Pb}^{4+}(12 \%)$ oxidation states. Tin (Fig. $2 \mathrm{i}$ ) is present as $\mathrm{Sn}^{2+}$ (76\%) and $\mathrm{Sn}^{4+}(24 \%)$, which justifies the presence of tin in both Qand $\mathrm{H}$-layers. Antimony (Fig. 2j) is present as $\mathrm{Sb}^{3+}(87 \%)$ and $\mathrm{Sb}^{5+}$ $(13 \%)$, whereas iron (Fig. $2 \mathrm{k}$ ) is present as $\mathrm{Fe}^{2+}$. Figure $2 \mathrm{j}$ also shows a very weak $\mathrm{O} 1 \mathrm{~s}$ peak which suggests there is only weak oxidation at the surface so that cylindrite is stable under ambient conditions. Sulfur is exclusively present in $S^{2-}$ state as shown in Fig. 2l. The slight underrepresentation of $\mathrm{Sb}$ and $\mathrm{Fe}$ in the derived formula may also be attributed to the overlap between the $\mathrm{Sb} 3 d$ peak with $\mathrm{O} 1 \mathrm{~s}$ peak, and Fe $2 p$ peak with $\mathrm{Sn} 3 p$ peak. The comparison between the XPS spectra of the pristine cylindrite crystal and the air-aged cylindrite crystal is shown in Supplementary Figure 2. It is observed that the XPS survey spectra of the pristine and air-aged crystals are just about identical and the surface stoichiometry of cylindrite remains almost intact. The amount of oxygen is further quantified by the peak fitting in the high-resolution spectra at the $\mathrm{Sb} 3 d$ and $\mathrm{O} 1 \mathrm{~s}$ binding energy region. Over three weeks of airaging, the atomic concentration ratio of $\mathrm{Sb}$ and $\mathrm{O}$ is only changed from 1:0.13 of the pristine crystal into 1:0.16. Therefore, it can be inferred that the effect of surface oxidation is very weak in cylindrite.

\section{Revealing structural anisotropy of cylindrite crystal}

The cylindrite thin flakes are mechanically exfoliated using Nitto tape onto a quartz substrate (details in the Methods section). Figure 3a shows a reflection optical microscope image of an exfoliated thin cylindrite crystal. The topography of the crystal surface is analyzed using atomic force microscopy (AFM) in Fig. 3b. From the height profile of the AFM image (inset of Fig. $3 b$ ), the thickness is determined to be $10 \mathrm{~nm}$ approximately which indicates that the flake consists of only 9 layer pairs. First, the in-plane structural anisotropy of the crystal is studied by angleresolved polarized Raman spectroscopy using a linearly polarized $632.8 \mathrm{~nm} \mathrm{He}-\mathrm{Ne}$ laser. Details of the experimental setup are described in the Methods section. Figure $3 c$ plots the parallel polarization component of the Raman spectra as a function of the linear polarization angle of the excitation laser. The Raman spectrum shows six distinct bands in the $50-350 \mathrm{~cm}^{-1}$ spectral region. The observed Raman peaks are located at 77, 90, 141, 182, 245 , and $312 \mathrm{~cm}^{-1}$. The Raman modes of cylindrite can be assigned according to the previously studied Raman spectra of the constituent components of $\mathrm{PbS}^{44}, \mathrm{SnS}^{45,46}, \mathrm{SnS}_{2}{ }^{47,48}, \mathrm{FeS}_{2}{ }^{49}$, and $\mathrm{Sb}_{2} \mathrm{~S}_{3}{ }^{50,51}$. The Raman peak at $77 \mathrm{~cm}^{-1}$ is attributed to the $A_{\mathrm{g}}$ mode of $\mathrm{Sb}_{2} \mathrm{~S}_{3}\left(73 \mathrm{~cm}^{-1}\right)$. The peak at $90 \mathrm{~cm}^{-1}$ is assigned as the $A_{g}$ mode of $\operatorname{SnS}\left(95 \mathrm{~cm}^{-1}\right)$. The $141 \mathrm{~cm}^{-1}$ peak corresponds to a superposition of phonon modes of both $\mathrm{SnS}_{2}$ lattices (the secondorder effects at $140 \mathrm{~cm}^{-1}$ ) and PbS lattices (transverse acoustic and optical modes at $\left.154 \mathrm{~cm}^{-1}\right)$. The peak at $182 \mathrm{~cm}^{-1}$ is most likely related to a combination of the $A_{g}$ mode of $\operatorname{SnS}\left(192 \mathrm{~cm}^{-1}\right)$ and the $A_{g}$ mode of $\mathrm{Sb}_{2} S_{3}\left(191 \mathrm{~cm}^{-1}\right)$. The peak at $245 \mathrm{~cm}^{-1}$ represents the vibrational mode of $\mathrm{Sb}_{2} \mathrm{~S}_{3}\left(238 \mathrm{~cm}^{-1}\right)$. In addition, the peak at $312 \mathrm{~cm}^{-1}$ is assigned as a combination of the $A_{g}$ mode of $\mathrm{Sb}_{2} \mathrm{~S}_{3}\left(312 \mathrm{~cm}^{-1}\right)$ and the $\mathrm{A}_{1 \mathrm{~g}}$ mode of $\mathrm{SnS}_{2}\left(315 \mathrm{~cm}^{-1}\right)$. It is noted that the Raman spectra of cylindrite are dominated by the contributions from $\mathrm{Sb}_{2} \mathrm{~S}_{3}, \mathrm{SnS}_{2}, \mathrm{SnS}$, and $\mathrm{PbS}$. The low energy and broad width of the Raman peaks are attributed to the superposition of the closely placed modes in the constituent lattices and the heavy damping present in the vdW heterostructure. Raman spectra of cylindrite flakes with other thicknesses are shown in Supplementary Figure 3. Almost no Raman shift is observed depending on the flake thickness due to the vdW stacking of the $\mathrm{H}$ - and Q-layers. From Fig. 3c, the intensity of each Raman mode in the parallel polarization configuration exhibits a strong dependence on the linear polarization angle of the incident 


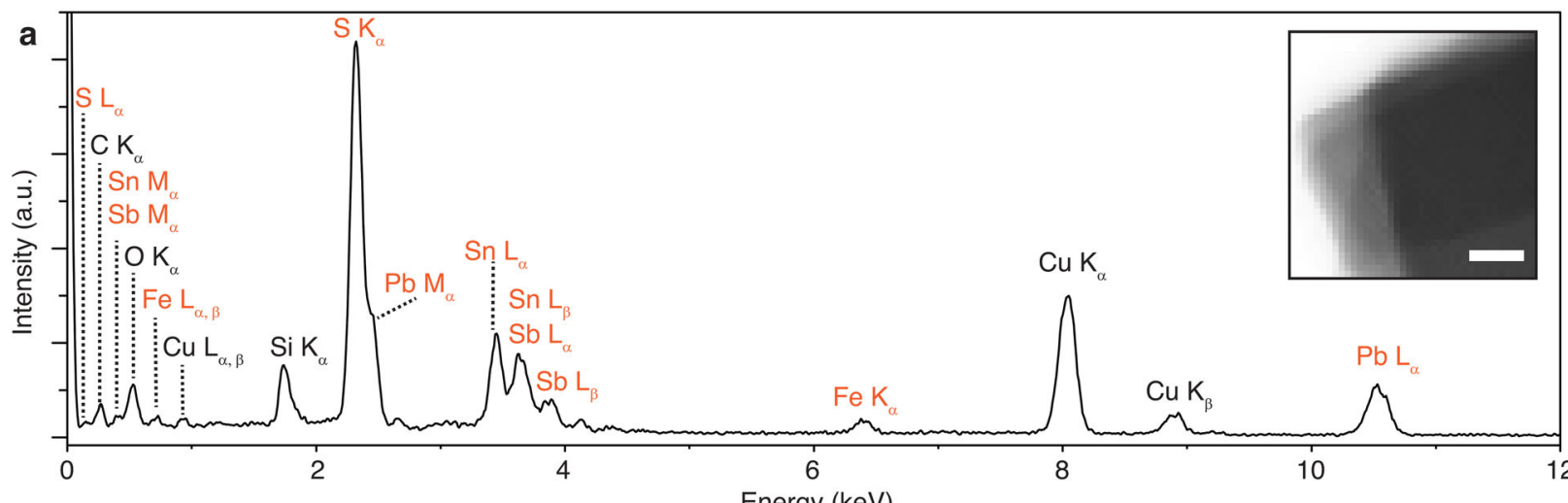

b
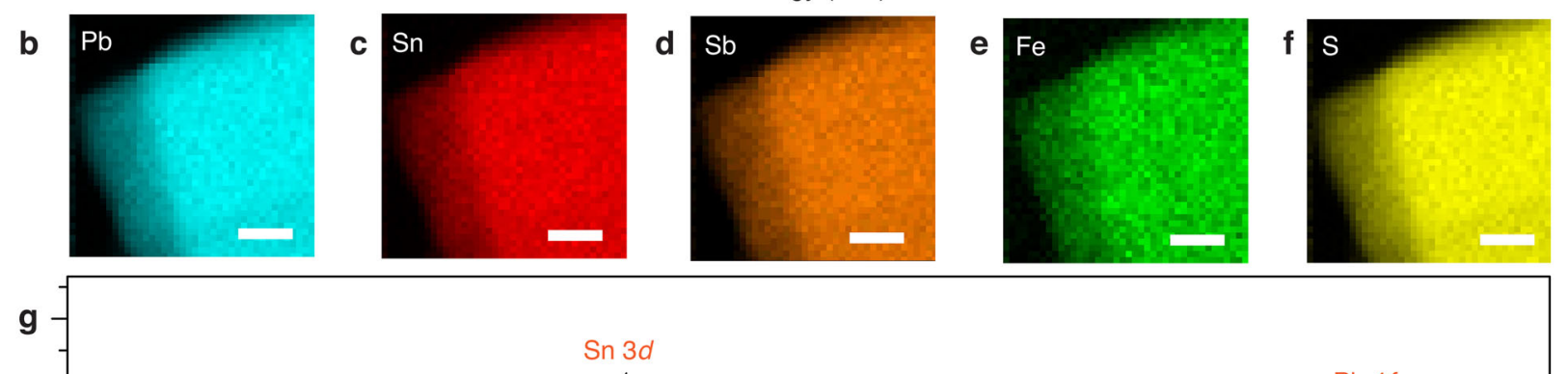

(1)
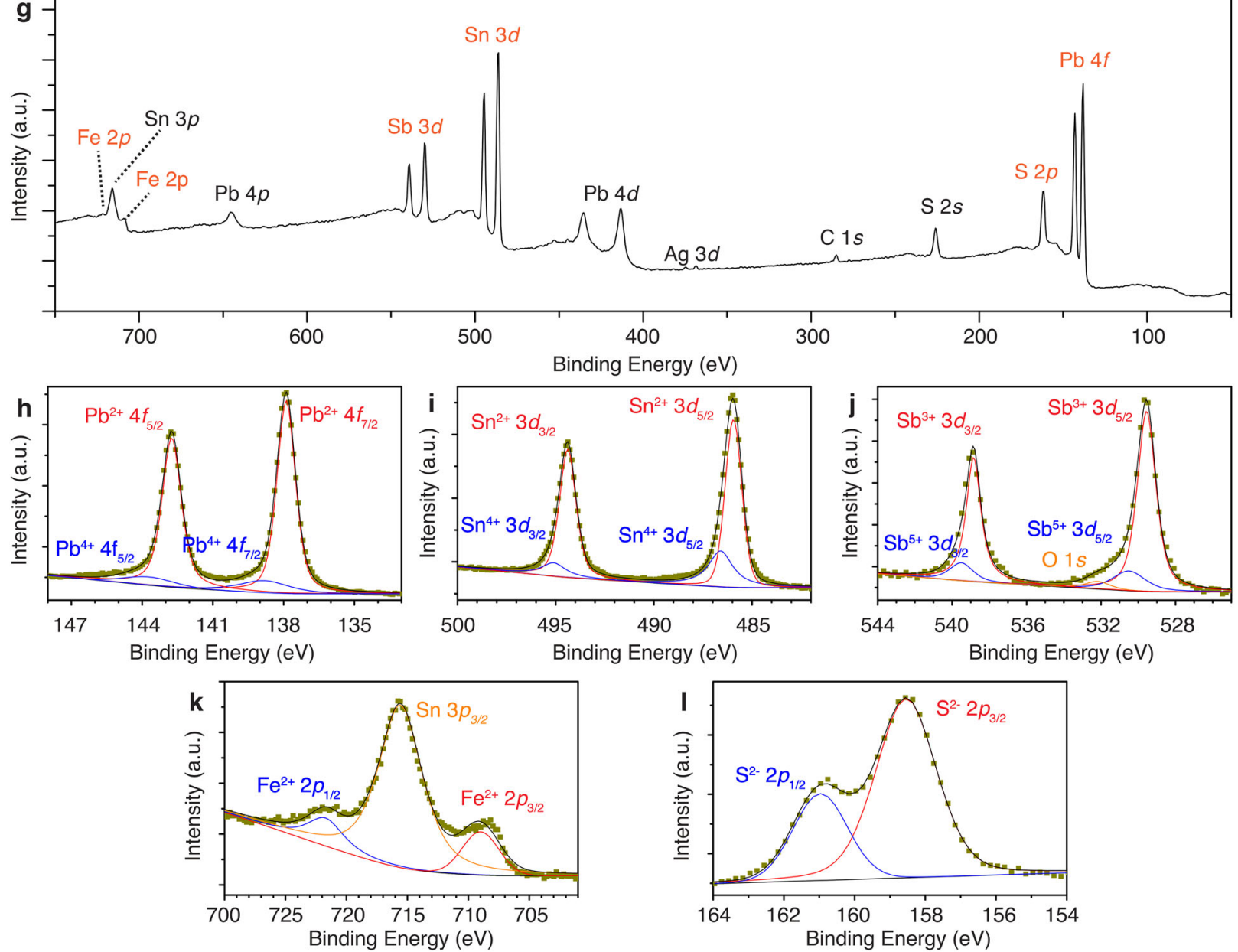

Fig. 2 Bulk and surface chemical composition of cylindrite. a Averaged EDXS spectrum from a thin cylindrite flake. The peaks of the five main elements $\mathrm{Pb}, \mathrm{Sn}, \mathrm{Sb}, \mathrm{Fe}$, and $\mathrm{S}$ labeled in orange color are used for the quantification in Table 1. b-f TEM-EDXS maps of the main elements $\mathrm{Pb}, \mathrm{Sn}, \mathrm{Sb}, \mathrm{Fe}$, and $\mathrm{S}$ from the area of a cylindrite crystal shown in the BF-TEM image in the inset of (a). The scale bar is $50 \mathrm{~nm}$. g Averaged XPS spectrum of cylindrite obtained from multiple measurements. The peaks labeled in orange color are used for the quantification in Table 2. h-I High-resolution spectra around the binding energy regions of $\mathrm{Pb} 4 f, \mathrm{Sn} 3 d, \mathrm{Sb} 3 d$, Fe $2 p$, and S $2 p$, respectively. 
beam. Such angular dependence of Raman intensity can be used to reveal the crystal structural anisotropy of cylindrite flakes and determine the crystal axes. Although the alternatively stacked $\mathrm{SnS}_{2}$-like and $\mathrm{PbS}$-like layers are isotropic, the incommensurability between these constituent 2D lattices introduces the straininduced deformation in the crystals, resulting in the anisotropic heterostructure superlattice. The Raman analysis reveals the structural anisotropy of such heterostructure due to the straininduced deformation. Figure $3 \mathrm{~d}-\mathrm{g}$ present the polar plots of the intensity variations of parallel polarization components for

Table 1. EDXS quantification of cylindrite.

\begin{tabular}{lc}
\hline Element & Average Concentration (at\%) \\
\hline $\mathrm{Pb}$ & $12: 30 \pm 0: 93$ \\
$\mathrm{Sn}$ & $16: 52 \pm 0: 48$ \\
$\mathrm{Fe}$ & $3: 93 \pm 0: 65$ \\
$\mathrm{Sb}$ & $7: 71 \pm 0: 78$ \\
$\mathrm{~S}$ & $59: 54 \pm 1: 84$ \\
\hline
\end{tabular}

Table 2. XPS quantification of cylindrite surface.

\begin{tabular}{lc}
\hline Element & Average Concentration (at\%) \\
\hline $\mathrm{Pb}$ & $14: 27 \pm 0: 29$ \\
$\mathrm{Sn}$ & $16: 45 \pm 1: 23$ \\
$\mathrm{Fe}$ & $3: 57 \pm 1: 04$ \\
$\mathrm{Sb}$ & $7: 75 \pm 0: 74$ \\
$\mathrm{~S}$ & $57: 96 \pm 1: 85$ \\
\hline
\end{tabular}

the Raman modes at $141,182,245$, and $312 \mathrm{~cm}^{-1}$ as a function of the incident linear polarization angle $\theta$. The triclinic crystal of cylindrite only supports $A_{g}$ modes, where the Raman tensor of a given $\mathrm{A}_{\mathrm{g}}$ mode is defined as $R=\left(\begin{array}{cc}u & w \\ w & v\end{array}\right)$, where $u, v$, and $w$ are the Raman tensor components. The parallel polarization component of the Raman $A_{g}$ modes can be expressed $a^{52}$,

$I_{\mathrm{A}_{g}}^{\|}(\theta) \propto\left(u \cos ^{2} \theta+2 w \sin \theta \cos \theta+v \sin ^{2} \theta\right)^{2}$

It is shown that the experimental measurements match well with the theoretical fits from Eq. (1). The anisotropic two-fold rotational symmetry of intensity is observed for each Raman mode with two unequal maxima as the incident linear polarization is along $0^{\circ}$ (x-direction) and $90^{\circ}$ (y-direction), where the $x$ - and $y$ directions corresponding to the crystal orientations are shown in Fig. 2a. The higher maximum at $\theta=0^{\circ}$ indicates that the $x$ direction corresponds to the rippling direction along the $c$-axis in the cylindrite crystal, whereas the $y$-direction is along the $b$-axis. Therefore, it can be inferred that the angle-resolved Raman mode analysis is a useful tool to show the crystal structural anisotropy and determine the crystal lattice orientations in cylindrite flakes.

\section{Linear dichroism in cylindrite thin flake}

To further explore the effect of the strain-induced lattice deformation on the anisotropic optical properties of the material, polarization-dependent DRS is performed on the cylindrite thin flakes, which is defined as,

$$
\mathrm{DRS}=\frac{R-R_{0}}{R_{0}}
$$

where the change in the reflectance $R$ from the thin flake is measured with respect to the reflectance $R_{0}$ from the bare quartz substrate and normalized by $R_{0}$. By definition, the magnitude of a
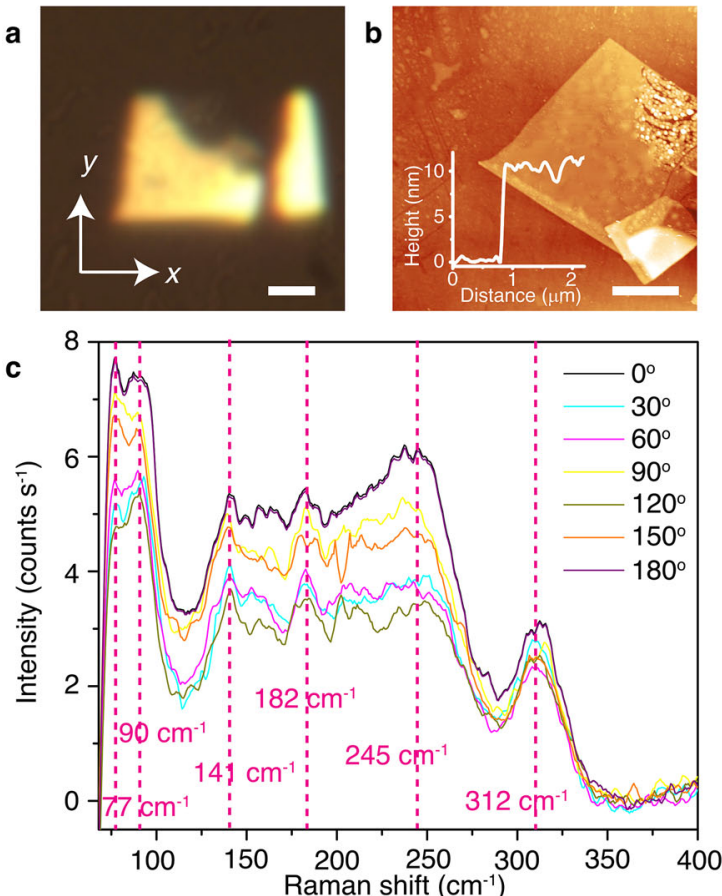

d
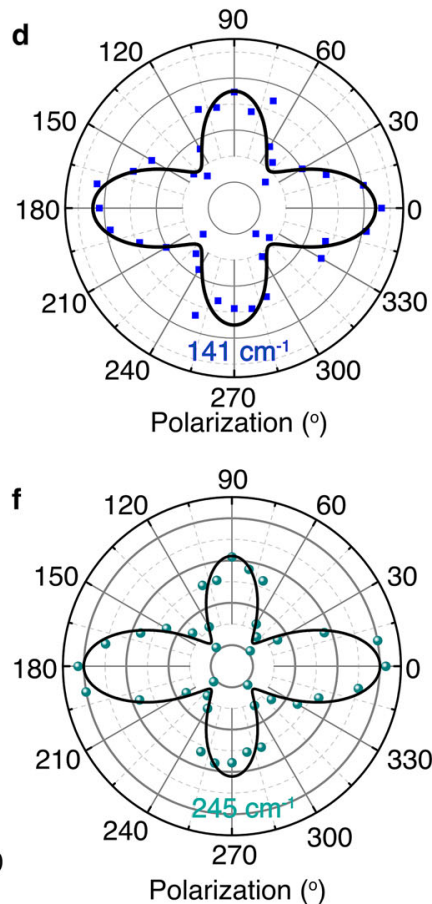

e
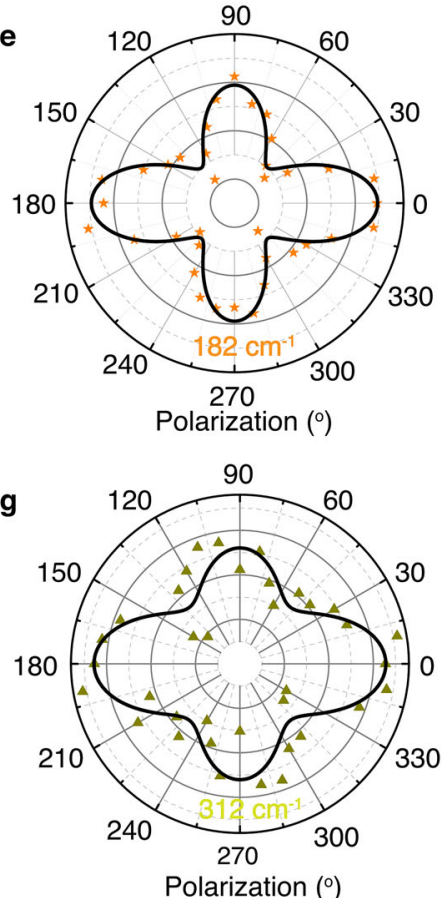

Fig. 3 In-plane structural anisotropy measured through Raman spectroscopy. a Reflection optical microscope image of an exfoliated cylindrite flake. $x$ - and $y$-axes signify reference axes. b AFM image of the cylindrite flake with the inset showing that the flake thickness is around $10 \mathrm{~nm}$. The scale bars are $2 \mu \mathrm{m}$. c Raman spectra acquired in the parallel polarization configuration from the same flake for different linear polarization angles between $0^{\circ}$ and $180^{\circ}$ with respect to the $x$-axis. $\mathbf{d}-\mathbf{g}$ Polar plots of the intensity variations of parallel polarization components for Raman modes at 141, 182, 245, and $312 \mathrm{~cm}^{-1}$. The data points are the experimentally measured values and the solid curves are the corresponding theoretical fits. 

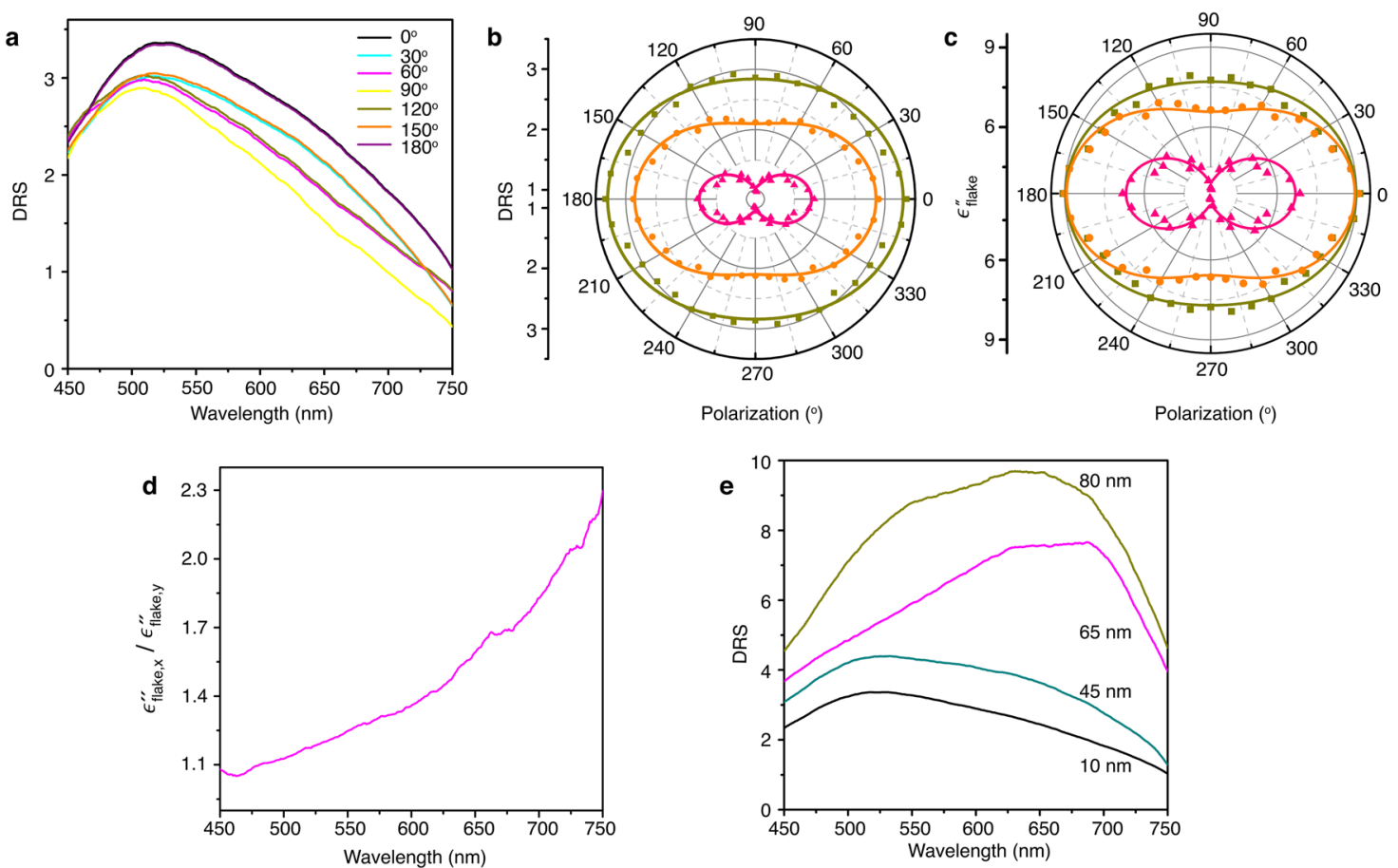

Fig. 4 Linear dichroism of cylindrite. a DRS spectra from the $10 \mathrm{~nm}$ thick cylindrite flake as a function of the incident polarization angle between $0^{\circ}$ and $180^{\circ}$. b, c Evolution of the measured DRS and the extracted $\epsilon_{\text {flake }}^{\prime \prime}$ as a function of the incident polarization angle for three different wavelengths of $520 \mathrm{~nm}$ (dark yellow), $600 \mathrm{~nm}$ (orange), and $700 \mathrm{~nm}$ (magenta). The measured data points are fitted with a sinusoidal function (solid curves). d $\epsilon_{\text {flake } x}^{\prime \prime} / \epsilon_{\text {flake.y } y}^{\prime \prime}$ response as a function of the excitation wavelength. e DRS spectra from cylindrite flakes of four different thicknesses $10,45,65$, and $80 \mathrm{~nm}$.

DRS is proportional to the absorption of the sample, and it can be easily linearized in the case of ultrathin flakes with thickness $d<<$ $\lambda$, with $\lambda$ being the wavelength of incident light ${ }^{53}$. For the normal incidence of light and transparent substrate such as quartz, DRS can be expressed as,

$\mathrm{DRS}=\frac{8 \pi d}{\lambda} \times \frac{\epsilon_{\text {flake }}^{\prime \prime}}{\epsilon_{\text {substrate }}^{\prime}-1}$

where $\epsilon=\epsilon^{\prime}+\mathrm{i} \epsilon^{\prime \prime}=(n+\mathrm{i} k)^{2}$ is the complex dielectric constant of the material with $\epsilon^{\prime}$ and $\epsilon^{\prime \prime}$ denoting the real and imaginary parts respectively, while $(n+i k)$ represents the complex refractive index. Therefore, DRS is directly proportional to the imaginary part $\epsilon_{\text {flake }}^{\prime \prime}$ of the cylindrite flake. Figure $4 a$ plots the acquired DRS spectra from the same $10 \mathrm{~nm}$ thick cylindrite flake in Fig. 3a for different incident linear polarization angles with respect to the rippling direction ( $x$-axis) of the crystal in the excitation wavelength range of $450-750 \mathrm{~nm}$. Details of the experimental setup are described in the Methods section. The polar plots in Fig. $4 \mathrm{~b}$ and $\mathrm{c}$ show the variations of the measured DRS and the extracted $\epsilon_{\text {flake }}^{\prime \prime}$ as a function of the incident linear polarization angle at three different wavelengths of 520,600 , and $720 \mathrm{~nm}$. A dipolar dependence is observed in all the cases. The DRS and $\epsilon_{\text {flake }}^{\prime \prime}$ data are further fitted with a sinusoidal function of the form $a_{x} \cos ^{2}$ $\theta+a_{y} \sin ^{2} \theta$, where $a_{x}$ and $a_{y}$ are constants proportional to the absorbance values as the incident white light is linearly polarized along $x$ - and $y$-axes respectively. It is indicated that the absorbance reaches the maximum when the incident linear polarization is along the rippling direction of the crystal (along the $c$-axis), while it is the minimum when the incident polarization is perpendicular to the rippling direction (along the $b$-axis). Therefore, it is evident that strong in-plane structural anisotropy resulted from the lattice deformation in cylindrite crystal gives rise to linear dichroism, even though the individual $\mathrm{H}$ - and Q-layers are not expected to exhibit any in-plane anisotropy. In
Fig. $4 \mathrm{~d}$, the linear dichroism is quantified by plotting the variation of the ratio $\epsilon_{\text {flake }, x}^{\prime \prime} / \epsilon_{\text {flake, } y}^{\prime \prime}$ between the values of $\epsilon_{\text {flake }}^{\prime \prime}$ as the incident polarization is along the rippling direction of the crystal and its perpendicular direction. It is observed that the anisotropic linear optical response increases gradually with the excitation wavelength. Figure $4 \mathrm{e}$ plots the measured DRS spectra of four individual cylindrite flakes of different thicknesses 10, 45, 65, and $80 \mathrm{~nm}$, when the incident white light is polarized along the rippling direction of each crystal. The crystal axes of each crystal are determined through angle-resolved polarized Raman spectroscopy measurements, which are shown in Supplementary Figure 4. A higher magnitude in DRS for a thicker crystal indicates that the absorption is more for a thicker sample. In addition, the redshift in the DRS spectrum as a function of thickness justifies a more yellowish glow in the reflection optical image of the thicker crystal compared to the greenish-yellow glow of a thinner one.

\section{Anisotropic THG response from cylindrite flakes}

The effect of in-plane structural anisotropy on the nonlinear optical properties of cylindrite crystal is investigated through character polarization-dependent anisotropic THG response. The details of the experimental setup are provided in the Methods section. When the $c, b$, and $a$ axes of the triclinic cylindrite crystal is oriented along $x, y$, and $z$ directions, the contracted form of its third-order nonlinear susceptibility tensor $\left(x^{(3)}\right)$ can be expressed as $^{54}$,

$X^{(3)}=\left[\begin{array}{llllllllll}X_{11} & X_{12} & X_{13} & X_{14} & X_{15} & X_{16} & X_{17} & X_{18} & X_{19} & X_{10} \\ X_{21} & X_{22} & X_{23} & X_{24} & X_{25} & X_{26} & X_{27} & X_{28} & X_{29} & X_{20} \\ X_{31} & X_{32} & X_{33} & X_{34} & X_{35} & X_{36} & X_{37} & X_{38} & X_{39} & X_{30}\end{array}\right]$

where the first subscript 1, 2, 3 represents $x, y, z$ respectively, and the second subscript refers to the following combination of three 
components as,

$\begin{array}{cccccccccc}x x x & y y y & z z z & y z z & y y z & x z z & x x z & x y y & x x y & x y z \\ 1 & 2 & 3 & 4 & 5 & 6 & 7 & 8 & 9 & 0\end{array}$

The electric field of the pump beam at frequency $\omega$ which is linearly polarized at an angle $\theta$ with respect to the $x$-axis can be written as $\overrightarrow{\mathbf{E}}^{(\omega)}=E_{0}(\cos \theta \hat{x}+\sin \theta \hat{y})$, where $\hat{x}$ and $\hat{y}$ are the unit vectors along the $x$ - and $y$-axis. Since the polarization of the excitation electric field always remains in the $x$-y plane in the current experimental configuration, no contribution from the components of $X^{(3)}$ containing $z$ terms will be observed in the THG signal, so that there are only eight nonzero elements of $X^{(3)}$ as $X_{11}$, $X_{12}, X_{18}, X_{19}, X_{21}, X_{22}, X_{28}$ and $X_{29}$. Since the electric field of THG is proportional to $X^{(3)}$, the $x$ - and $y$-polarized components of the THG output intensity can be expressed as,

$$
\begin{aligned}
& I_{x}^{3(\omega)} \propto\left(X_{11} \cos ^{3} \theta+x_{12} \sin ^{3} \theta+3 X_{18} \cos \theta \sin ^{2} \theta+3 X_{19} \sin \theta \cos ^{2} \theta\right)^{2} \\
& I_{y}^{3(\omega)} \propto\left(X_{21} \cos ^{3} \theta+X_{22} \sin ^{3} \theta+3 X_{28} \cos \theta \sin ^{2} \theta+3 X_{29} \sin \theta \cos ^{2} \theta\right)^{2}
\end{aligned}
$$

The inset of Fig. 5a shows the transmission optical microscope image of the same $10 \mathrm{~nm}$ thick flake of Fig. 3a, where the green THG signal from the flaking area is observed as it is excited by a $1560 \mathrm{~nm}$ pump laser with the spot size of $1.5 \mu \mathrm{m}$. The THG spectrum in Fig. 5a shows the expected peak emission wavelength at $520 \mathrm{~nm}$, which is exactly one-third of the fundamental wavelength. Figure $5 \mathrm{~b}$ shows the log-scale plot of the THG power as a function of the pump power, where the cubic power-law dependence further confirms the THG process. For the average pump power of $4 \mathrm{~mW}$ corresponding to a peak pump irradiance of $27.2 \mathrm{GW} \mathrm{cm}^{-2}$, the obtained THG conversion efficiency is around $2 \times 10^{-9}$ for the $10 \mathrm{~nm}$ thick flake. Next, the effect of the incident linear polarization angle on the THG emission power from the cylindrite crystal is characterized. The desired linear polarization of the pump beam is obtained by placing a linear polarizer oriented along the $x$-axis (parallel to the rippling direction of the flake) and a rotating half-wave plate. Figure $5 c-f$ plot the angular dependence of the THG emission power as a function of incident linear polarization angle with respect to the $x$-axis for four cylindrite flakes with thicknesses of $10,45,80$, and $150 \mathrm{~nm}$. The black and magenta data points are the measured $x$ - and $y$-polarized components of the THG power, while the dark cyan data points show the measured total THG power. The solid lines in Fig. 5c are theoretical fits of the experimental data using Eq. (5), showing a good agreement between the theoretical prediction and the experimental measurements. The optical images, the angle-resolved polarized Raman characterization for determining the crystal orientation, and the corresponding anisotropic THG characterization for the cylindrite flakes of different thicknesses are included in Supplementary Figure 4. It is evident that the polarization-dependent THG emission from the cylindrite crystal exhibits an anisotropic two-fold rotational symmetry, with the maximum THG emission occurring at the incident linear polarization along $0^{\circ}$ (parallel to the rippling direction along the $c$-axis) and the second maximum THG emission along $90^{\circ}$ (perpendicular to the rippling direction along the $b$-axis). The evolution of the anisotropy ratio of the THG signal $l_{x}^{(3 \omega)}(\theta=0) / l_{y}^{(3 \omega)}\left(\theta=90^{\circ}\right)$ between the incident polarizations parallel and perpendicular to the rippling direction as a function of the flake thickness is plotted in Fig. $5 \mathrm{~g}$. It is shown that for all the cylindrite flakes, the anisotropy ratio almost keeps as a constant with an average value of $2.33 \pm 0.19$. The structural anisotropy in the cylindrite crystal is further reflected in the extracted relative magnitudes of the $x^{(3)}$ tensor. The relative magnitudes of the anisotropic $X^{(3)}$ tensor elements can be extracted from Eq. (5). It is found that the values of $x^{(3)}$ tensor elements stay almost unchanged for cylindrite flakes of different thicknesses, with the average relative magnitudes as $X_{11}: X_{12}$ : $X_{18}: X_{19}: X_{21}: X_{22}: X_{28}: X_{29}=1: 0.012: 0.121: 0.016: 0.003:$ $0.663: 0.002: 0.107$, which further reveals the intrinsic nature of the anisotropic nonlinear optical response of the cylindrite crystal.

The air-stability of the $10 \mathrm{~nm}$ thick cylindrite flake is investigated by Raman spectroscopy and THG characterization after keeping the flake under ambient conditions for four weeks. The acquired Raman spectrum from the air-aged cylindrite flake is plotted in Supplementary Figure 5, which is almost exactly the same as that of the freshly exfoliated flake in Fig. 3c, indicating all the Raman modes are observed to be intact after air-aging. The comparison between the THG characterization of the freshly exfoliated flake and that of the air-aged one is presented in Supplementary Figure 6. It is observed that the THG emission power and THG conversion efficiency remain at the same level as the freshly exfoliated flake, even after four weeks of air-aging. In addition, the shape of the polarization-dependent anisotropic THG emission pattern remains intact after air-aging, with almost the same anisotropy ratio of the THG signal. Hence, it can be inferred that the exfoliated cylindrite flakes are stable in air.

Moreover, the dependence of the THG response as a function of the cylindrite flake thickness is investigated. It is possible to extract the magnitude of $X^{(3)}$ from the following equation for the average power of THG emission $P^{(3 \omega)}$, which is derived by solving nonlinear Maxwell's equations as ${ }^{34}$,

$$
P^{(3 \omega)}(d)=\frac{9 \omega^{2} d^{2}}{16 \sqrt{n_{3}^{2}+k_{3}^{2}} n_{1}^{3} \epsilon_{0}^{2} c^{4}}\left|x^{(3)}\right|^{2} \frac{P^{(\omega) 3}}{f_{\text {rep }}^{2} W^{4} \tau^{2}\left[\frac{\pi}{4 \ln 2}\right]^{3}}\left(\frac{e^{-\frac{4 \pi k_{3} d}{\lambda_{3}}}-2 \cos (\Delta k d) e^{-\frac{2 \pi k_{3} d}{\lambda_{3}}}+1}{d^{2}\left(\frac{4 \pi^{2} k_{3}^{2}}{\lambda_{3}^{2}}+\Delta k^{2}\right)}\right) e^{-\frac{4 n_{3} d}{\lambda_{3}}}
$$

where $d$ is the thickness of the cylindrite flake, $n_{1}$ and $n_{3}$ are the real part of the refractive index at the fundamental frequency $(\omega)$ and THG frequency $(3 \omega), k_{3}$ is the imaginary part of the refractive index at $3 \omega$, and $\Delta k=\frac{6 \pi}{\lambda_{1}}\left(n_{1}-n_{3}\right)$ is the phase mismatch between the forward propagating fundamental beam and the thirdharmonic emission beam in the transmission microscope arrangement. The parameters of the Gaussian fundamental pulsed laser include average pump power $P^{(\omega)}$, beam spot size $W$, pulse width $\tau$, and repetition rate $f_{\text {rep. }}$.

The black data points in Fig. 5h show the measured average THG power as a function of the cylindrite flake thickness at the pump power of $1.5 \mathrm{~mW}$ when the incident linear polarization is parallel to the rippling direction ( $x$-axis). It is clearly shown that within the range of thickness less than $60 \mathrm{~nm}$, the THG power increases quadratically up to around $8 \mathrm{pW}$ as the flake gets thicker, giving the maximum conversion efficiency of $5.33 \times 10^{-9}$. However, for the thickness greater than $60 \mathrm{~nm}$, exponential decay of the THG signal is observed. This is attributed to the significant attenuation of the THG signal as it propagates through the flake due to the strong absorption of the THG signal in the cylindrite flake especially with large thickness. Therefore, by fitting the measured data with an exponentially decaying function (black curve) of the form $P^{(3 \omega)}(d)=C d^{2} \exp \left(-\frac{4 \pi k_{3} d}{\lambda_{3}}\right)$ with $C$ as a constant, the imaginary part of refractive index at $\lambda_{3}=520 \mathrm{~nm}$ for the incident linear polarization along the $x$-axis is extracted as $k_{3 x}=1.45$. The real part of the refractive index along the $x$-axis can then be estimated from the measured $\epsilon_{\text {flake } x}^{\prime \prime}=2 n_{3 x} k_{3 x}$ (Fig. 4c) as $n_{3 x}=3.12$. These extracted refractive index values for cylindrite are consistent with the measured ones for franckeite. Since cylindrite is having a similar chemical composition and layered structure as franckeite, it is expected to have a refractive index within the same range of franckeite. Next, the $x_{11}^{(3)}$ corresponding to the magnitude of $\chi^{(3)}$ for the $x$-polarized pump beam is extracted from the fitting in Eq. (6) by using the estimated values of $n_{3 x}$ and $k_{3 x}$. Taking into account the pump laser parameters of $P^{(\omega)}=1.5 \mathrm{~mW}, \tau=90 \mathrm{fs}$, $f_{\text {rep }}=80 \mathrm{MHz}$, and $W=$ 

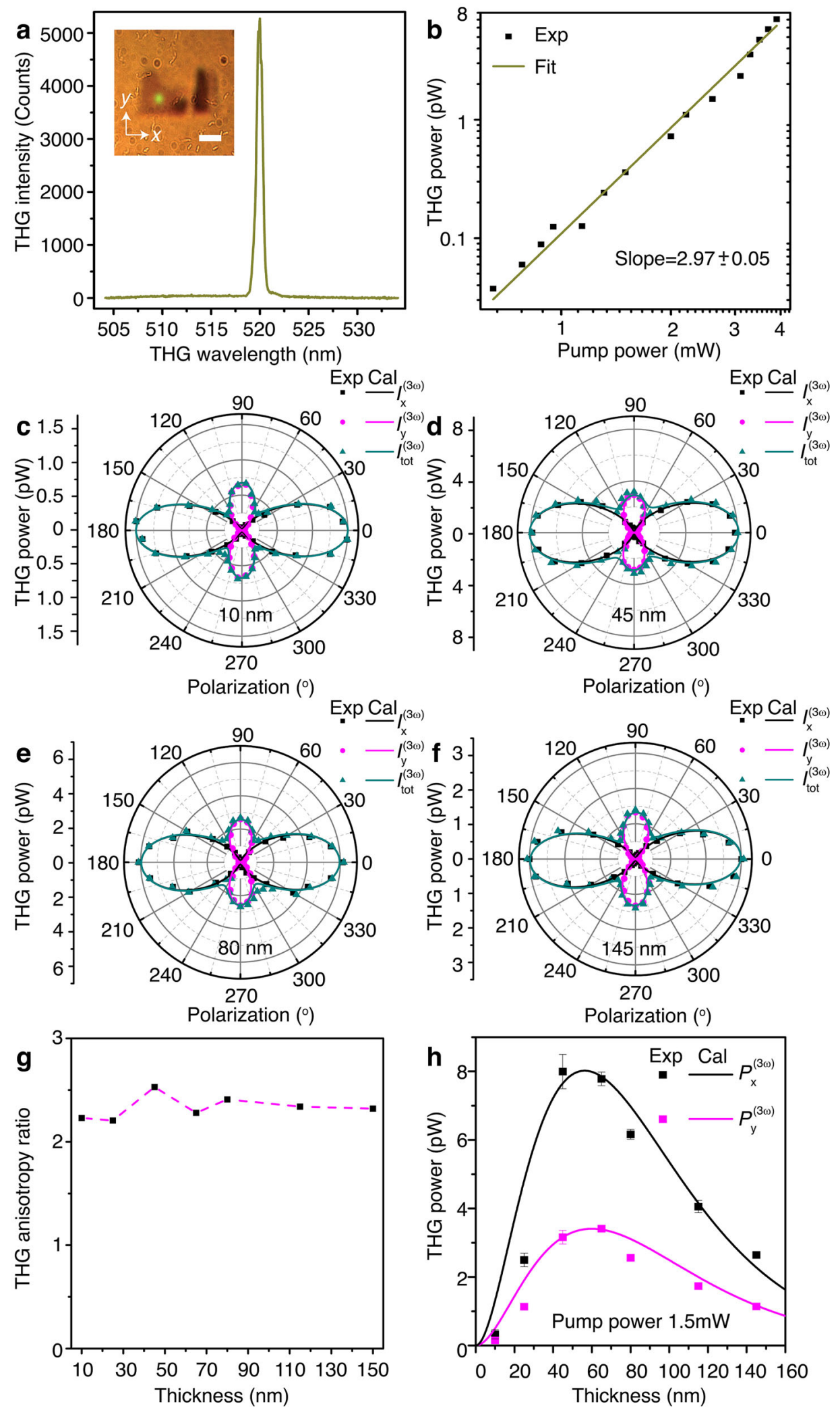

Fig. 5 Anisotropic THG response from cylindrite flakes. a The measured spectrum of THG emission with a peak wavelength at $520 \mathrm{~nm}$ from the $10 \mathrm{~nm}$ thick cylindrite flake, which is exactly one-third of the fundamental wavelength of the pump beam at $1560 \mathrm{~nm}$. Inset shows a transmission microscope image of THG emission from the flake. The scale bar is $2 \mu \mathrm{m}$. $\mathbf{b}$ The double log-scale plot of the measured THG emission power as a function of the pump power. c-f Angular dependence of the THG emission power as a function of incident linear polarization angle of the pump beam for four cylindrite flakes with the thicknesses of $10,45,80$, and $150 \mathrm{~nm}$. $0^{\circ}$ corresponds to the $x$-axis along the rippling direction of the crystal. $\mathbf{g}$ Measured THG anisotropy ratio as a function of the flake thickness. $\mathbf{h}$ Evolution of the average THG emission power versus the flake thickness for incident linear polarization along the $x$-axis (black) and $y$-axis (magenta). 


\begin{tabular}{|c|c|c|c|c|c|c|}
\hline Cylindrite & Triclinic & $10-60$ & 1560 & $2.1-2.5$ & 3.06 & This work \\
\hline BP & Orthorhombic & 30 & 1560 & $\sim 3.5$ & 1.6 & 33 \\
\hline $\operatorname{ReS}_{2}$ & Triclinic & $0.73-10$ & 1515 & $\sim 1.5$ & 35 & 36 \\
\hline SiP & Orthorhombic & $10-40$ & 1560 & $1.6-2.1$ & 1.8 & 55 \\
\hline
\end{tabular}

$1.5 \mu \mathrm{m}$ at $\lambda_{1}=1560 \mathrm{~nm}$ with $n_{1}=4$, the magnitude of $\chi_{11}^{(3)}$ is estimated to be $3.06 \times 10^{-19} \mathrm{~m}^{2} \mathrm{~V}^{-2}$. In Fig. $5 \mathrm{~h}$, the magenta data points are the evolution of the measured THG power with flake thickness for the incident linear polarization perpendicular to the rippling direction ( $y$-axis). Again, by fitting the THG power data with an exponentially decaying function and using the measured $\epsilon_{\text {flake, } y}^{\prime \prime}=2 n_{3 y} k_{3 y}$, the refractive index values $k_{3 y}$ and $n_{3 y}$ along $y$ axis can be estimated as 1.38 and 2.79 , respectively. The extracted magnitude of $x_{22}^{(3)}$ representing $x^{(3)}$ for $y$-polarized pump beam is $2.33 \times 10^{-19} \mathrm{~m}^{2} \mathrm{~V}^{-2}$, which gives the $X_{11}^{(3)} / \chi_{22}^{(3)}$ ratio of 1.31 for the cylindrite crystal. Finally, to assess the THG response in cylindrite, the measured THG anisotropy ratio and $x^{(3)}$ value of the cylindrite crystal are compared with the already reported values of other existing anisotropic nonlinear 2D materials including $B P, \operatorname{ReS}_{2}$, $\mathrm{GeSe}, \mathrm{GeAs}$ and SiP, which are tabulated in Table 3. It shows that cylindrite has a strong nonlinear optical response with the thirdorder nonlinear susceptibility almost double that of BP.

\section{DISCUSSION}

In conclusion, we have demonstrated in-plane structural anisotropy and highly anisotropic optical responses of the cylindrite crystal, where two individual $\mathrm{SnS}_{2}$-like and $\mathrm{PbS}$-like isotropic layers are stacked to create the natural vdW heterostructure. The vdW interaction, local atomic alignment, and lattice deformation while stacking of the constituent $\mathrm{SnS}_{2}$-like and PbS-like layers create small out-of-plane rippling on the crystal surface, resulting in strong inplane structural anisotropy in the crystal. It is shown that the direction of the ripples and hence the in-plane structural anisotropy in the crystal can be probed by angle-resolved polarized Raman spectroscopy. The effect of the strong structural anisotropy on the linear and nonlinear optical properties of the cylindrite crystal is further studied. Through polarization-dependent DRS measurements, it is revealed that cylindrite thin flakes exhibit strong linear dichroism, which is not only important in the context of understanding the origin of optical anisotropy in natural vdW heterostructures but also can be harnessed to design future polarization-sensitive photodetectors and transistors. Moreover, polarization-dependent anisotropic THG responses from cylindrite thin flakes is investigated. It is shown that the THG emission power as the pump beam is linearly polarized along the rippling direction of the crystal (c-axis) is more than two times compared to that along the perpendicular direction ( $b$-axis). The relative magnitudes of the eight nonzero components of the $x^{(3)}$ tensor are extracted as an estimate of the nonlinear optical anisotropy in the crystal. Lastly, it is shown that the THG emission power is highly dependent on the flake thickness, where the THG response is maximum around the flake thickness of $60 \mathrm{~nm}$. The estimated $x^{(3)}$ of cylindrite crystal is found to be $3.06 \times 10^{-19} \mathrm{~m}^{2} \mathrm{~V}^{-2}$, indicating that cylindrite has a strong nonlinear optical response as other anisotropic nonlinear $2 \mathrm{D}$ materials such as BP. Additionally, unlike BP, cylindrite is stable in ambient conditions, providing an added advantage for practical use in building future on-chip anisotropic nonlinear optical devices for optical information processing and communication.

\section{METHODS}

\section{Sample preparation}

The quartz substrate is sonicated in deionized water, acetone, and isopropanol one after the other. The cylindrite thin flakes are mechanically exfoliated from the bulk natural cylindrite mineral (from Poopó town, Oruro, Bolivia) using Nitto tape (SPV 224). Then the flakes are transferred directly onto the quartz substrate by placing the tape containing the flakes in contact to the quartz surface and peeling it off fast. Now, flakes of different thicknesses on the quartz substrate can be readily identified by monitoring their optical transparency under a transmission optical microscope. Flakes with lower thickness exhibit higher optical transparency. Finally, the thickness of each identified flake is confirmed by AFM measurement.

For the TEM measurement, exfoliated cylindrite flakes are transferred from the quartz substrate to TEM copper grids following polymethyl methacrylate (PMMA)-assisted wet transfer method. First, the quartz substrate containing the thin flakes is spin-coated $(2000 \mathrm{rpm}, 60 \mathrm{~s})$ with PMMA ( $950 \mathrm{kDa}$ ) and baked at $120^{\circ} \mathrm{C}$ for $2 \mathrm{~min}$ to facilitate the adhesion between cylindrite and PMMA. Next, the sample is immersed in $3 \mathrm{M}$ potassium chloride $(\mathrm{KOH})$ solution and kept at $50^{\circ} \mathrm{C}$ for $1.5-2 \mathrm{~h}$. After quartz etching by $\mathrm{KOH}$, the PMMA film containing the cylindrite crystals is washed with deionized (DI) water two times for $10 \mathrm{~min}$ at each step to remove the residual $\mathrm{KOH}$. Finally, the PMMA film is fished out of the DI water on a TEM grid. The TEM grid covered with the PMMA film is left uncovered for drying out naturally. Finally, the PMMA is washed out by dissolving it in acetone while the crystals are transferred on the TEM grid.

\section{Experimental setup}

For analyzing the Raman spectrum, a $632.8 \mathrm{~nm}$ He-Ne laser beam is passed through a linear polarizer and a half-wave plate and then focused on the cylindrite flake using a $40 \times$ objective lens $(N A=0.65)$. The back-reflected light is collected by the same objective lens and directed towards a spectrometer (Horiba, iHR 520) using a beam splitter. The excitation laser is blocked by placing a Rayleigh rejection filter (Semrock, LP02-633RE-25) in front of the spectrometer. The parallel polarization component of the Raman spectrum is analyzed using another linear polarizer before the spectrometer.

For the polarization-resolved DRS measurement, light from a broadband white light source (Thorlabs, SLS201L, $360-2600 \mathrm{~nm}$ ) is passed through a linear polarizer and focused on the cylindrite flake using a $100 \times$ objective lens $(\mathrm{NA}=0.7)$. The reflection spectrum $(R)$ is obtained by collecting the back-reflected light from the sample using the same objective lens and directing it towards the spectrometer with a beam splitter. An iris is used to spatially filter out a small area of the flake. Then the flake is removed from the collection area and the reflection spectrum from the quartz substrate $\left(R_{0}\right)$ is measured. Finally, DRS is calculated as DRS $=\frac{R-R_{0}}{R_{0}}$.

For the THG measurements, the pump laser beam at the fundamental wavelength of $1560 \mathrm{~nm}$ (Calmar fiber laser, pulse width $90 \mathrm{fs}$, repetition rate $80 \mathrm{MHz}$ ) is transmitted through a linear polarizer and a rotating halfwave plate to set the incident linear polarization angle, and then focused onto the cylindrite flake using a $40 \times$ objective lens $(N A=0.65)$. The transmitted THG emission from the cylindrite flake is collected by a $100 \times$ objective lens $(\mathrm{NA}=0.7)$, filtered spectrally with a short pass filter (Thorlabs, FESH 900) to remove the transmitted fundamental pump beam, 
and then focused onto a color charge-coupled device (CCD) camera. For the spectral characterization of the THG emission, a spectrometer (Horiba, iHR 520) is used instead of the CCD camera.

\section{DATA AVAILABILITY}

The data that support the findings of this study are available from the corresponding author upon reasonable request.

Received: 1 April 2021; Accepted: 30 July 2021; Published online: 20 August 2021

\section{REFERENCES}

1. Xia, F., Wang, H., Xiao, D., Dubey, M. \& Ramasubramaniam, A. Two-dimensional material nanophotonics. Nat. Photonics 8, 899-907 (2014).

2. Wang, Q. H., Kalantar-Zadeh, K., Kis, A., Coleman, J. N. \& Strano, M. S. Electronics and optoelectronics of two-dimensional transition metal dichalcogenides. Nat. Nanotechnol. 7, 699-712 (2012).

3. Fiori, G. et al. Electronics based on two-dimensional materials. Nat. Nanotechnol. 9, 768-779 (2014)

4. Geim, A. K. \& Grigorieva, I. V. Van der Waals heterostructures. Nature 499, 419-425 (2013).

5. Novoselov, K. S., Mishchenko, A., Carvalho, A. \& Castro Neto, A. H. 2D materials and van der Waals heterostructures. Science 353, aac9439 (2016).

6. Ribeiro-Palau, R. et al. Twistable electronics with dynamically rotatable heterostructures. Science 361, 690-693 (2018).

7. Chen, W. et al. Direct observation of van der Waals stacking-dependent interlayer magnetism. Science 366, 983-987 (2019).

8. Withers, F. et al. Light-emitting diodes by band-structure engineering in van der Waals heterostructures. Nat. Mater. 14, 301-306 (2015).

9. Liu, Y. et al. Van der Waals heterostructures and devices. Nat. Rev. Mater. 1, 16042 (2016).

10. Wang, C. Y. et al. Gate-tunable van der Waals heterostructure for reconfigurable neural network vision sensor. Sci. Adv. 6, eaba6173 (2020).

11. Lyu, J., Pei, J., Guo, Y., Gong, J. \& Li, H. A new opportunity for 2D van der Waals heterostructures: making steep-slope transistors. Adv. Mater. 32, 1906000 (2020).

12. Shin, G. H. et al. High-performance field-effect transistor and logic gates based on GaS-MoS2 van der Waals heterostructure. ACS Appl. Mater. Interfaces 12, 5106-5112 (2020).

13. Massicotte, M. et al. Picosecond photoresponse in van der Waals heterostructures. Nat. Nanotechnol. 11, 42-46 (2016).

14. De Fazio, D. et al. High responsivity, large-area Graphene/MoS 2 flexible photodetectors. ACS Nano 10, 8252-8262 (2016).

15. Ye, L., Li, H., Chen, Z. \& Xu, J. Near-infrared photodetector based on $\mathrm{MoS}_{2} /$ black phosphorus heterojunction. ACS Photonics 3, 692-699 (2016).

16. Sun, X. et al. Tunable ultrafast nonlinear optical properties of graphene/MoS 2 van der Waals heterostructures and their application in solid-state bulk lasers. ACS Nano 12, 11376-11385 (2018)

17. Cai, Z., Liu, B., Zou, X. \& Cheng, H. M. Chemical vapor deposition growth and applications of two-dimensional materials and their heterostructures. Chem. Rev. 118, 6091-6133 (2018).

18. Gong, Y. et al. Vertical and in-plane heterostructures from $\mathrm{WS}_{2} / \mathrm{MoS}_{2}$ monolayers. Nat. Mater. 13, 1135-1142 (2014).

19. Shi, J. et al. All chemical vapor deposition synthesis and intrinsic bandgap observation of MoS2/Graphene heterostructures. Adv. Mater. 27, 7086-7092 (2015).

20. Uwanno, T., Hattori, Y., Taniguchi, T., Watanabe, K. \& Nagashio, K. Fully dry PMMA transfer of graphene on $\mathrm{h}-\mathrm{BN}$ using a heating/cooling system. 2D Mater. 2, 041002 (2015)

21. Kretinin, A. V. et al. Electronic properties of Graphene encapsulated with different two-dimensional atomic crystals. Nano Lett. 14, 3270-3276 (2014)

22. Haigh, S. J. et al. Cross-sectional imaging of individual layers and buried interfaces of graphene-based heterostructures and superlattices. Nat. Mater. 11, 764-767 (2012).

23. Wang, X. \& Xia, F. Stacked 2D materials shed light. Nat. Mater. 14, 264-265 (2015).

24. Niu, Y. et al. Mechanical and liquid phase exfoliation of cylindrite: a natural van der Waals superlattice with intrinsic magnetic interactions. 2D Mater. 6, 035023 (2019).

25. Velický, M. et al. Exfoliation of natural van der Waals heterostructures to a single unit cell thickness. Nat. Commun. 8, 14410 (2017)
26. Chen, $\mathrm{H}$. et al. A Natural $2 \mathrm{D}$ heterostructure $\left[\mathrm{Pb}_{3.1} \mathrm{Sb}_{0.9} \mathrm{~S}_{4}\right]\left[\mathrm{Au}_{\mathrm{x}} \mathrm{Te}_{2-\mathrm{x}}\right]$ with large transverse nonsaturating negative magnetoresistance and high electron mobility. J. Am. Chem. Soc. 141, 7544-7553 (2019).

27. Frisenda, R. et al. Symmetry breakdown in franckeite: spontaneous strain, rippling, and interlayer moiré. Nano Lett. 20, 1141-1147 (2020).

28. Molina-Mendoza, A. J. et al. Franckeite as a naturally occurring van der Waals heterostructure. Nat. Commun. 8, 14409 (2017).

29. Li, J. et al. Nonlinear optical response in natural van der Waals heterostructures. Adv. Opt. Mater. 8, 2000382 (2020).

30. Sun, Z., Martinez, A. \& Wang, F. Optical modulators with 2D layered materials. Nat. Photonics 10, 227-238 (2016).

31. Autere, A. et al. Nonlinear optics with 2D layered materials. Adv. Mater. 30, 1705963 (2018).

32. Eggleton, B. J. Nonlinear optics in 2D materials. APL Photonics 4, 034101 (2019).

33. Autere, A. et al. Rapid and large-area characterization of exfoliated black phosphorus using third-harmonic generation microscopy. J. Phys. Chem. Lett. 8, 1343-1350 (2017).

34. Youngblood, N., Peng, R., Nemilentsau, A., Low, T. \& Li, M. Layer-tunable thirdharmonic generation in multilayer black phosphorus. ACS Photonics 4, 8-14 (2017).

35. Hipolito, F. \& Pedersen, T. G. Optical third harmonic generation in black phosphorus. Phys. Rev. B 97, 035431 (2018)

36. Cui, Q., Muniz, R. A., Sipe, J. E. \& Zhao, H. Strong and anisotropic third-harmonic generation in monolayer and multilayer ReS $_{2}$. Phys. Rev. B 95, 165406 (2017).

37. Dasgupta, A., Gao, J. \& Yang, X. Anisotropic third-harmonic generation in layered Germanium Selenide. Laser Photonics Rev. 14, 1900416 (2020).

38. Sar, H., Gao, J. \& Yang, X. In-plane anisotropic third-harmonic generation from germanium arsenide thin flakes. Sci. Rep. 10, 14282 (2020).

39. Yin, X. et al. Edge nonlinear optics on a $\mathrm{MoS}_{2}$ atomic monolayer. Science 344, 488 (2014).

40. Li, Y. et al. Probing symmetry properties of few-layer $\mathrm{MoS}_{2}$ and h-BN by optical second-harmonic generation. Nano Lett. 13, 3329-3333 (2013).

41. Makovicky, E., Petřičcek, V., Dušek, M. \& Topa, D. Crystal structure of a synthetic tinselenium representative of the cylindrite structure type. Am. Mineralogist 93, 1787-1798 (2008).

42. Meinecke, C., Vogt, J., Kaden, R., Bente, K. \& Butz, T. Investigation of synthetic cylindrite-microstructures. Nucl. Instrum. Methods Phys. Res. B: Beam Interact. Mater. At. 260, 317-320 (2007).

43. Kaden, R. et al. Synthesis and physical properties of cylindrite micro tubes and lamellae. Phys. Status Solidi B 247, 1335-1350 (2010).

44. Smith, G. D., Firth, S., Clark, R. J. H. \& Cardona, M. First- and second-order Raman spectra of galena (PbS). J. Appl. Phys. 92, 4375-4380 (2002).

45. Chandrasekhar, H. R., Humphreys, R. G., Zwick, U. \& Cardona, M. Infrared and Raman spectra of the IV-VI compounds SnS and SnSe. Phys. Rev. B 15, 2177-2183 (1977).

46. Li, M. et al. Revealing anisotropy and thickness dependence of Raman spectra for SnS flakes. RSC Adv. 7, 48759-48765 (2017).

47. Smith, A. J., Meek, P. E. \& Liang, W. Y. Raman scattering studies of $\mathrm{SnS}_{2}$ and $\mathrm{SnSe}_{2}$. J. Phys. C: Solid State Phys. 10, 1321-1323 (1977).

48. Mead, D. G. \& Irwin, J. C. Raman spectra of $\mathrm{SnS}_{2}$ and $\mathrm{SnSe}_{2}$. Solid State Commun. 20, 885-887 (1976).

49. Vogt, H., Chattopadhyay, T. \& Stolz, H. J. Complete first-order Raman spectra of the pyrite structure compounds $\mathrm{FeS}_{2}, \mathrm{MnS}_{2}$, AND SiP 2 . J. Phys. Chem. Solids 44, 869-873 (1983).

50. Parize, R. et al. In situ analysis of the crystallization process of $\mathrm{Sb}_{2} \mathrm{~S}_{3}$ thin films by Raman scattering and X-ray diffraction. Mater. Des. 121, 1-10 (2017).

51. Sereni, P. et al. Polarization-Dependent Raman Characterization of Stibnite $\left(\mathrm{Sb}_{2} \mathrm{~S}_{3}\right)$. AIP Conf. Proc. 1267, 1131-1132 (2010).

52. Hart, L., Dale, S., Hoye, S., Webb, J. L. \& Wolverson, D. Rhenium dichalcogenides: layered semiconductors with two vertical orientations. Nano lett. 16, 1381-1386 (2016).

53. Forker, R. \& Fritz, T. Optical differential reflectance spectroscopy of ultrathin epitaxial organic films. Phys. Chem. Chem. Phys. 11, 2142-2155 (2009).

54. Yang, X. L. \& Xie, S. W. Expression of third-order effective nonlinear susceptibility for third-harmonic generation in crystals. Appl. Opt. 34, 6130-6135 (1995).

55. Sar, H., Gao, J. \& Yang, X. 2D layered SiP as anisotropic nonlinear optical material. Sci. Rep. 11, 6372 (2021).

\section{ACKNOWLEDGEMENTS}

The authors acknowledge support from the National Science Foundation under Grant No. ECCS-1653032 and DMR-1552871. The authors also thank Shatadru Chakravarty, Brian Porter and Eric Bohannan for their help in acquiring the TEM, XPS and AFM data. 


\section{AUTHOR CONTRIBUTIONS}

X.Y., J.G. and A.D. conceived the idea of the research. A.D. performed the experiments. X.Y. and J.G. directed the research. All authors discussed the results and contributed to the manuscript.

\section{COMPETING INTERESTS}

The authors declare no competing interests.

\section{ADDITIONAL INFORMATION}

Supplementary information The online version contains supplementary material available at https://doi.org/10.1038/s41699-021-00254-9.

Correspondence and requests for materials should be addressed to J.G. or X.Y.

Reprints and permission information is available at http://www.nature.com/ reprints
Publisher's note Springer Nature remains neutral with regard to jurisdictional claims in published maps and institutional affiliations.

Open Access This article is licensed under a Creative Commons Attribution 4.0 International License, which permits use, sharing, adaptation, distribution and reproduction in any medium or format, as long as you give appropriate credit to the original author(s) and the source, provide a link to the Creative Commons license, and indicate if changes were made. The images or other third party material in this article are included in the article's Creative Commons license, unless indicated otherwise in a credit line to the material. If material is not included in the article's Creative Commons license and your intended use is not permitted by statutory regulation or exceeds the permitted use, you will need to obtain permission directly from the copyright holder. To view a copy of this license, visit http://creativecommons. org/licenses/by/4.0/.

(c) The Author(s) 2021 